\title{
Hemostatic agent use in thyroid surgery: a meta-analysis
}

\author{
Helmi Khadra ${ }^{1}$, Mohamed Bakeer ${ }^{2}$, Adam Hauch $^{1}$, Tian Hu ${ }^{3}$, Emad Kandil $^{1}$ \\ ${ }^{1}$ Division of Endocrine and Oncologic Surgery, Department of Surgery, Tulane University School of Medicine, New Orleans, LA, USA; ${ }^{2}$ Department \\ of Surgery, Louisiana State University School of Medicine, New Orleans, LA, USA; ${ }^{3}$ Tulane University School of Public Health and Tropical \\ Medicine, New Orleans, LA, USA \\ Contributions: (I) Conception and design: H Khadra, E Kandil; (II) Administrative support: E Kandil; (III) Provision of study materials or patients: A \\ Hauch; (IV) Collection and assembly of data: H Khadra, M Bakeer; (V) Data analysis and interpretation: T Hu; (VI) Manuscript writing: All authors; \\ (VII) Final approval of manuscript: All authors. \\ Correspondence to: Emad Kandil, MD, MBA, FACS, FACE. Department of Surgery, Tulane University School of Medicine, 1430 Tulane Ave., New \\ Orleans, LA 70112, USA. Email: Ekandil@tulane.edu.
}

Background: The use of hemostatic agents in thyroid surgery has been widely reported in the literature. The aim of this study is to assess the safety and efficacy of hemostatic agents in comparison to conventional techniques for hemostasis by meta-analysis of the current literature.

Methods: Articles were identified from PubMed and EMBASE using the following keyword searches: "hemostatic agent and thyroid surgery" and "hemostatic agent and thyroidectomy". Outcomes included total operative time, estimated blood loss, length of hospital stay, and intraoperative and postoperative complications. Data were extracted following review of appropriate studies by two independent authors and outcome differences were calculated using analysis of variance and the Bonferroni method.

Results: Thirty-four publications were identified and 10 studies met our inclusion criteria, totaling 941 patients, 519 (55.1\%) of which received a hemostatic agent during thyroid surgery. Of these patients who had hemostatic agents, $369(71 \%)$ received a hemostatic gel and $150(29 \%)$ received an oxidized cellulose patch. Outcome measures in each of these groups were compared with the patients receiving only conventional methods of hemostasis. The risk of hematoma formation in the hemostatic gel group was comparable to conventional hemostatic methods (95\% CI: 0.33, 2.59). This was also true when comparing conventional hemostasis to the patch (95\% CI: $0.64,15.24)$. No difference in the risk for seroma formation was found between the conventional and hemostatic gel groups (95\% CI: $0.26,3.95$ ). Drain output was significantly less in the gel group $40.75 \pm 35.6 \mathrm{~mL}$ compared to $66.26 \pm 31.2 \mathrm{~mL}$ in the conventional group (95\% CI: -23.422, -7.460). Patients who received hemostatic agents had shorter hospital stays when compared to the conventional group (95\% CI: $-1.057,-0.203$ ).

Conclusions: Our meta-analysis suggests that the use of a hemostatic agent in thyroid surgery yields minimal advantages for the management of perioperative bleeding risk.

Keywords: Hemostatic agent; thyroidectomy; thyroid surgery; hemostasis

Submitted Feb 22, 2018. Accepted for publication Feb 27, 2018.

doi: $10.21037 /$ gs.2018.03.02

View this article at: http://dx.doi.org/10.21037/gs.2018.03.02

\section{Introduction}

Thyroid surgery remains as the primary treatment modality in various thyroid pathologies (1). The first surgical approaches for the treatment of thyroid disorders were associated with inadmissibly high rates of mortality and morbidity due to hemorrhage, asphyxiation, air embolism, and infection (2). Implementation and standardization of the capsular dissection technique in 1973 by Thompson and colleagues (3) and of the technique of precise ligation of the arterial blood supply for performing a meticulous dissection of the thyroid gland decreased the morbidity 
and mortality associated with thyroid surgery to less than $1 \%$ (3-5). Nevertheless, postoperative hematoma remains an uncommon but potentially life-threaten complication of thyroid surgery. Post-thyroidectomy hemorrhage has a reported incidence in the literature of between $0.5 \%$ and $4.3 \%$ (6). The conventional techniques for hemostasis are suture ligation, electrocautery, or surgical clips. Difficulties in the control of oozing-type bleeding may also obscure adjacent anatomical structures and may cause inadvertent injuries to the RLN and parathyroid glands. Over the last two decades, a number of adjuvant hemostatic agents have been developed for hemostasis in thyroid surgery (7-9).

These agents have been broadly classified into three groups: topical hemostats which cause blood to clot at a bleeding surface, sealants which prevent leakage from tissues including vessels, and adhesives which bond tissues $(10,11)$. There is overlap among these categories, and many compounds can be classified into multiple groups. Topical hemostats are the most commonly used and typically consist of a mechanical surface to promote clot formation often with either thrombin or fibrinogen or a combination thereof. In 1998, the Food and Drug Administration approved the first fibrin sealant in the United States (11). Since then, a wide range of hemostatic agents have received approval, often for a narrow spectrum of surgical procedures after the demonstration of safety $(10,11)$. The aim of this study is to assess the safety and efficacy of hemostatic agents in comparison to conventional techniques for hemostasis by meta-analysis of the current literature.

\section{Methods}

\section{Identification of trials and data extraction}

Articles published through June 2015 on PubMed, EMBASE, and the Cochrane Database of Systematic Review were systematically searched by two independent reviewers using the following medical subject headings (MeSH): "Hemostatic Agent and Thyroid Surgery" and "Hemostatic Agent and Thyroidectomy". Additionally, all articles included in the analysis underwent reference review for other potential articles. The inclusion criteria for eligibility were as follows: (I) articles comparing thyroid surgery and the use of hemostatic agents; (II) studies that reported outcomes of hematoma formation, seroma formation, drain output, incidence of infection, recurrent laryngeal nerve injury, transient hypocalcemia, length of hospital stay, and age; (III) prospective studies, retrospective studies, controlled clinical trials, or randomized controlled trials; and (IV) studies that reported a measure of variance (standard error, standard deviation, or confidence interval). Articles not reported in English were excluded. For research groups with redundant patient populations, the latest study on that population was included for analysis. Results from the two independent reviewers were compared for accuracy, with disagreement resolved by consensus.

\section{Statistical analysis}

Primary outcomes of this study included operative time, hematoma formation, seroma formation, infection, RLN injury, transient hypocalcemia, drain output, length of hospital stay, and age. Odds ratios were calculated for categorical outcomes, while mean net changes were calculated for continuous variables. DerSimonian and Laird random-effects models were used to pool mean net changes or odds ratios across the studies (12). Funnel plots were constructed in order to assess publication bias and Begg's rank correlation test was used to examine the asymmetry of these plots. Egger's weighted linear regression test was used to examine the association between the mean effect estimate and its variance. Additionally, sensitivity analyses were conducted by excluding each study in turn, to evaluate their relative influence on the pooled estimates. All analyses were conducted in STATA (version 10; College Station, Texas, USA).

\section{Results}

Searching the three databases, thirty-four publications were identified. Nine studies were removed because hemostatic agents were not used in the studies; agents such as Ultrasonic Dissector, Focus, Harmonic, and Ligasure were used instead. Four studies were eliminated because the studies did not evaluate hemolytic agents. In the end, ten studies met inclusion criteria and underwent full text review for inclusion in the final analysis (Table 1).

In the ten studies, a total 941 patients were evaluated, of which $519(55.1 \%)$ received a hemostatic agent during a thyroid surgery. Of these patients who had hemostatic agents, 369 (71\%) received a hemostatic gel and 150 (29\%) received an oxidized cellulose patch. Outcome measures in each of these groups were compared with the patients receiving only conventional methods of hemostasis.

\section{Operative time}

Three studies analyzed the difference in operative times 
Table 1 Description of studies included in meta-analysis

\begin{tabular}{lcccc}
\hline Author & Year & Country & Study design & Sample (n) \\
\hline Testini et al. (8) & 2009 & Italy & Retrospective \\
Lachachi et al. (9) & 2000 & France & Prospective \\
Guler et al. (13) & 2011 & Turkey & Prospective \\
Sözen et al. (14) & 2011 & Turkey & Prospective \\
Kim et al. (15) & 2012 & Korea & Prospective \\
Ujam et al. (16) & 2012 & UK & Retrospective \\
Mathews et al. (17) & 1991 & USA & Retrospective \\
Patel et al. (18) & 2006 & USA & Prospective \\
Amit et al. (19) & 2013 & Israel & Prospective \\
Uwiera et al. (20) & 2005 & Austria & 100 \\
\hline
\end{tabular}

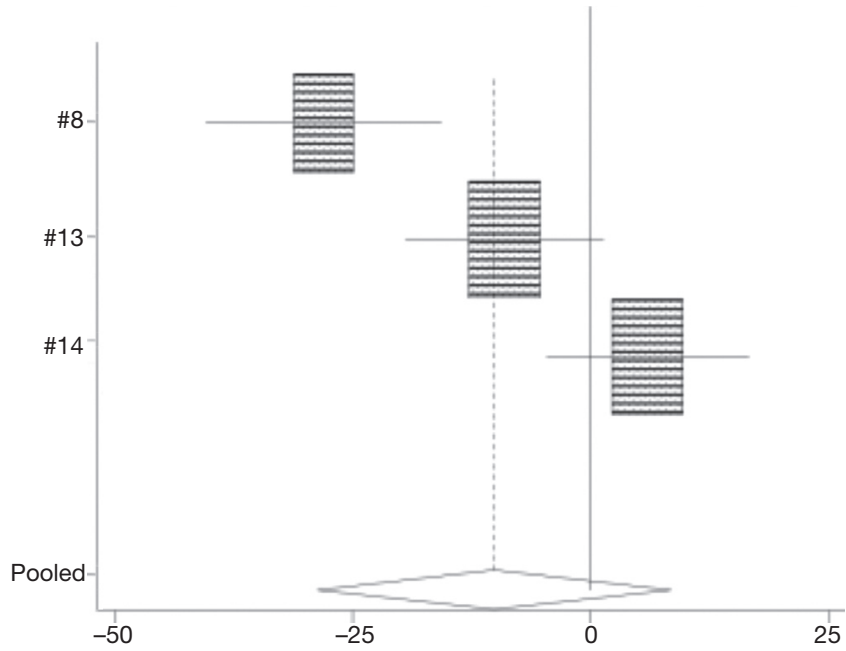

Net change in otime between $\mathrm{C}$ and $\mathrm{A}(95 \% \mathrm{Cl})$

Figure 1 Comparison of mean operative time of conventional $v s$. hemostatic gel.

with the use of hemostatic agents and conventional hemostatic approaches $(8,13,14)$. The mean operative time in the conventional group $114.95 \pm 21.8$ was comparable to the operative time in patients who received hemostatic gel $113.75 \pm 14.5$ minutes (95\% CI: $-28.634,8.427$ ) (Figure 1). Only Testini et al. (8) found a significant difference in shorter operative time with the use of hemostatic gel $v s$. conventional hemostasis. Two studies looked at the difference between conventional hemostasis and the hemostatic patch $(8,13)$. Patients who received oxidized cellulose patch had the shortest operative time,

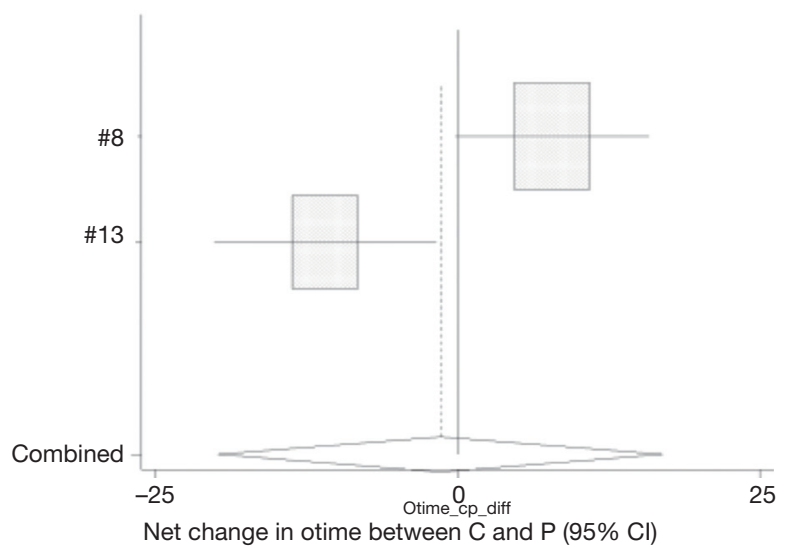

Figure 2 Mean operative time of conventional $v s$. hemostatic patch.

$107.3 \pm 20.7$ min (95\% CI: $-19.881,16.962)$ (Figure 2). Only Testini et al. (8) found a significant difference between the patch and conventional hemostasis.

\section{Hematoma formation}

Six studies compared the difference between hemostatic gel and conventional hemostatic methods on the risk of hematoma formation $(8,13-15,17,18)$. The risk of hematoma formation in the hemostatic gel group was comparable to conventional hemostatic methods $(95 \%$ CI: $0.33,2.59)$ (Figure 3). None of the studies reviewed found a significant difference between the two groups on hematoma formation. Two studies analyzed the difference between the hemostatic patch and conventional hemostatic 


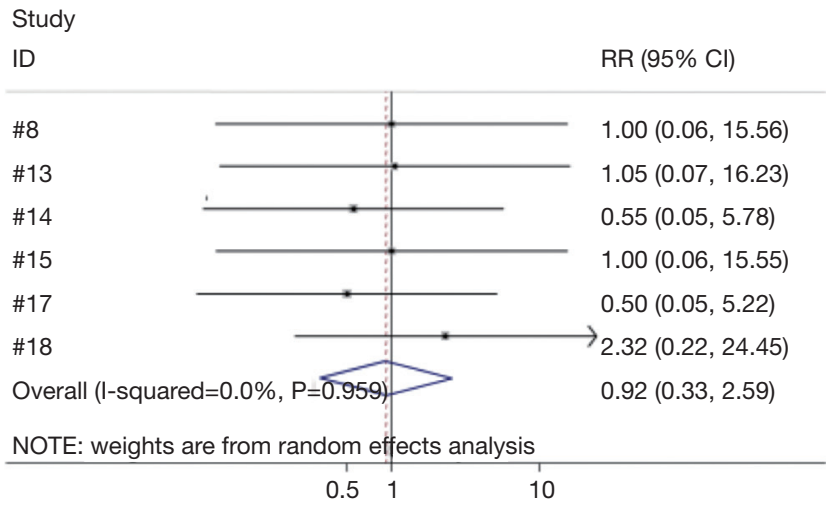

Figure 3 Risk of hematoma with conventional vs. hemostatic gel.

\section{Study}

ID

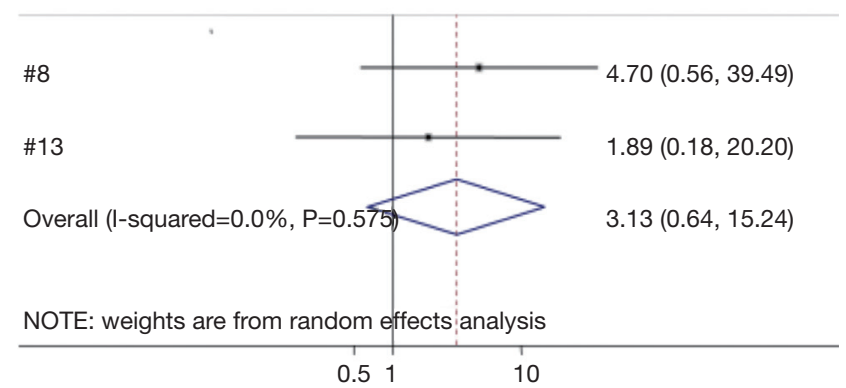

Figure 4 Risk of hematoma with conventional vs. hemostatic patch.

methods $(8,13)$. Again no significant difference was found when comparing conventional hemostasis to the patch $(95 \%$ CI: $0.64,15.24$ ) (Figure 4). Neither of the studies found a significant difference between the two groups.

\section{Seroma formation}

Three studies analyzed the effect of conventional methods for hemostasis and hemostatic gel on seroma formation $(14,15,17)$. No difference in the risk for seroma formation was found between the conventional and hemostatic gel groups (95\% CI: 0.26, 3.95) (Figure 5). None of the studies reviewed found a significant difference between conventional hemostasis methods and hemostatic gel use.

\section{Infection}

Two studies investigated the effect of hemostatic agents

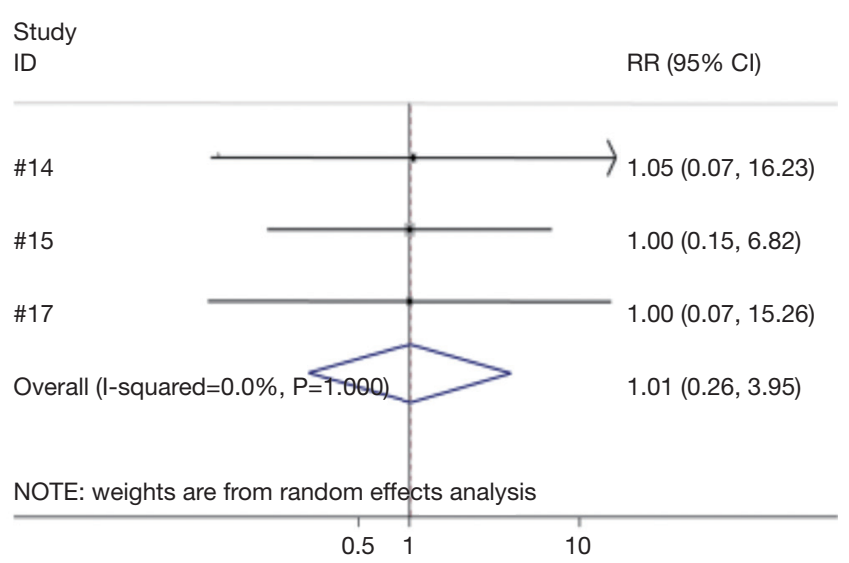

Figure 5 Risk of seroma formation with conventional and hemostatic gel.

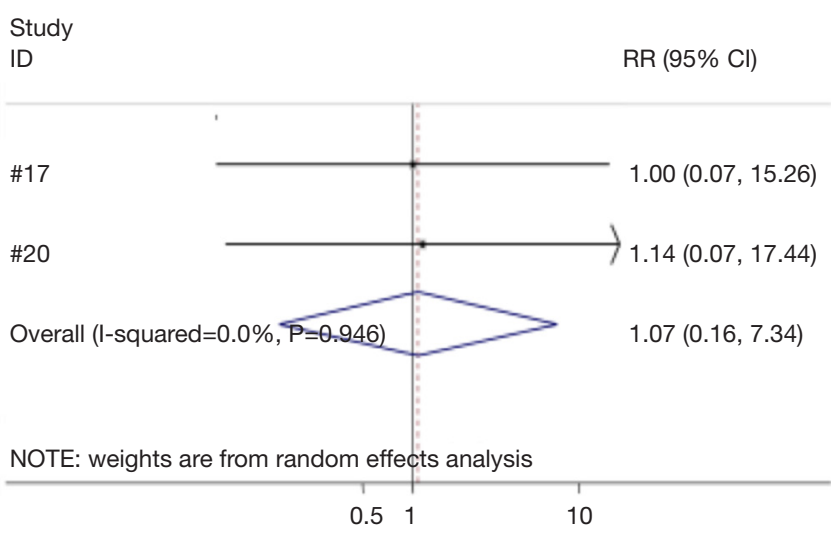

Figure 6 Risk of post-operative infection with conventional and hemostatic gel.

and conventional hemostatic methods on the risk of postoperative infection $(17,20)$. No difference in the risk for infection was found between the conventional and hemostatic gel groups (95\% CI: 0.16, 7.34) (Figure 6). Neither study reviewed found a significant difference between conventional hemostasis and hemostatic gel use.

\section{Recurrent laryngeal nerve injury}

Four studies compared the difference between hemostatic gel and conventional hemostatic methods on the risk of recurrent laryngeal nerve injury $(8,13,15,20)$. The risk of recurrent laryngeal nerve injury in the hemostatic gel group was comparable to conventional hemostatic methods (95\% CI: $0.20,2.47$ ) (Figure 7). None of the studies reviewed found a significant difference between the two groups on 


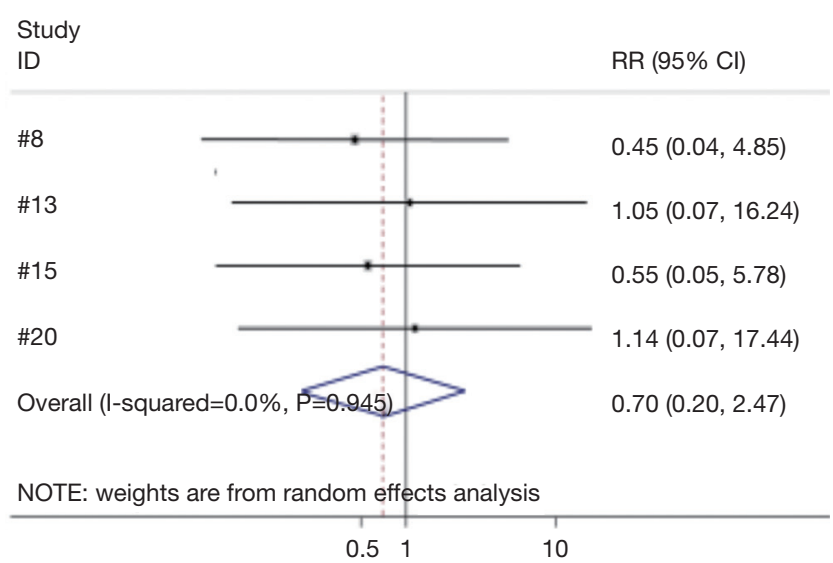

Figure 7 Risk of recurrent laryngeal nerve injury with conventional $v s$. hemostatic gel.

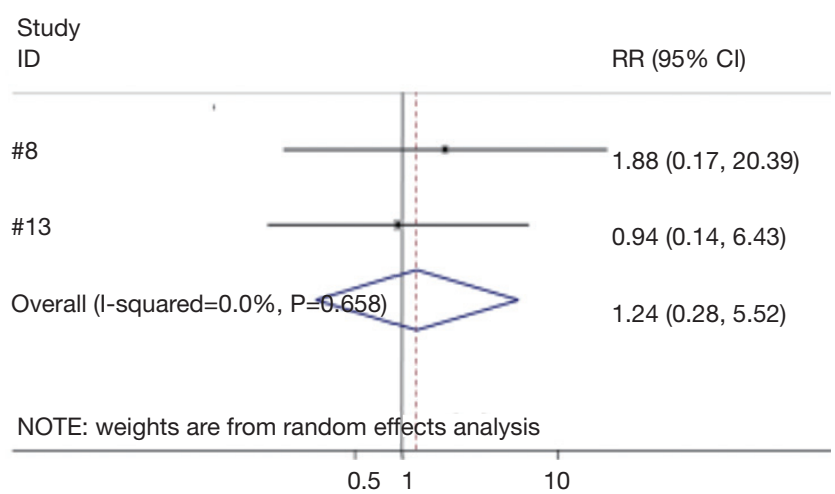

Figure 8 Risk of recurrent laryngeal nerve injury with conventional $v s$. hemostatic patch.

recurrent laryngeal nerve injury. Two studies analyzed the difference between the hemostatic patch and conventional hemostatic methods $(8,13)$. Again no significant difference was found when comparing conventional hemostasis to the patch (95\% CI: 0.28, 5.52) (Figure 8). Neither of the studies found a significant difference between the two groups.

\section{Transient hypoparathyroidism/bypocalcemia}

Four studies compared the difference between hemostatic gel and conventional hemostatic methods on the risk of hypoparathyroidism and hypocalcemia $(8,13,14,20)$. The risk of hypoparathyroidism in the hemostatic gel group was comparable to conventional hemostatic methods (95\% CI: 0.54, 1.54) (Figure 9). None of the studies reviewed found a significant difference between

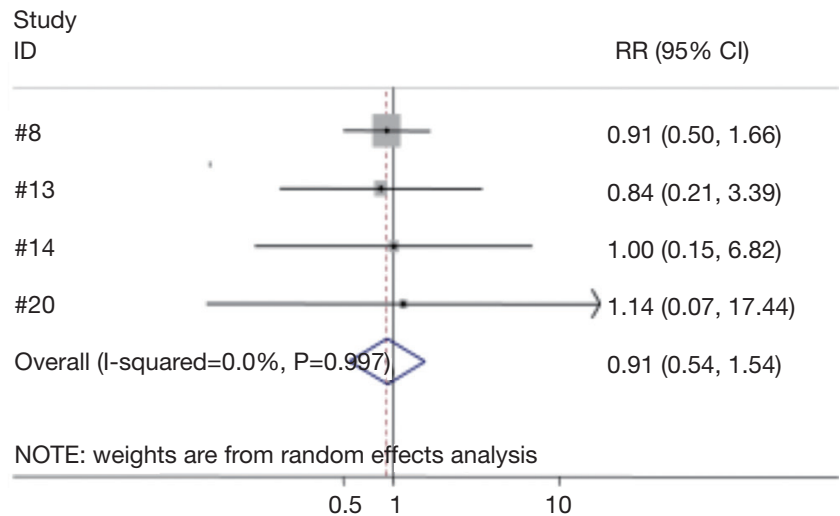

Figure 9 Risk of hyperparathyroidism/hypocalcemia with conventional $v s$. hemostatic gel.

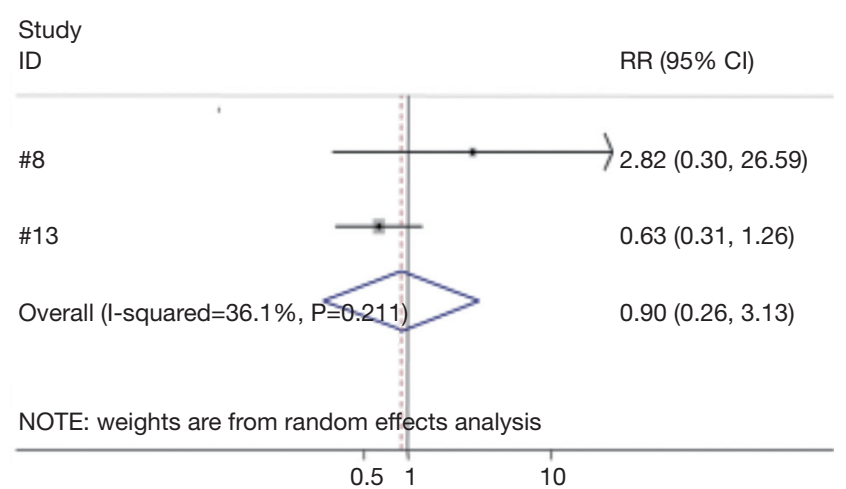

Figure 10 Risk of hyperparathyroidism/hypocalcemia with conventional vs. hemostatic patch.

the two groups on hypoparathyroidism. Two studies analyzed the difference between the hemostatic patch and conventional hemostatic methods $(8,13)$. Again no significant difference was found when comparing conventional hemostasis to the patch (95\% CI: 0.26, 3.13) (Figure 10). Neither of the studies found a significant difference between the two groups.

\section{Drain output}

Two studies investigated the effect of hemostatic agents and conventional hemostasis on drain output volume $(13,15)$. Drain output was significantly less in the gel group $40.75 \pm 35.6 \mathrm{~mL}$ compared to $66.26 \pm 31.2 \mathrm{~mL}$ in the conventional group (95\% CI: -23.422, -7.460) (Figure 11). Only one study (13) found a significant difference between 


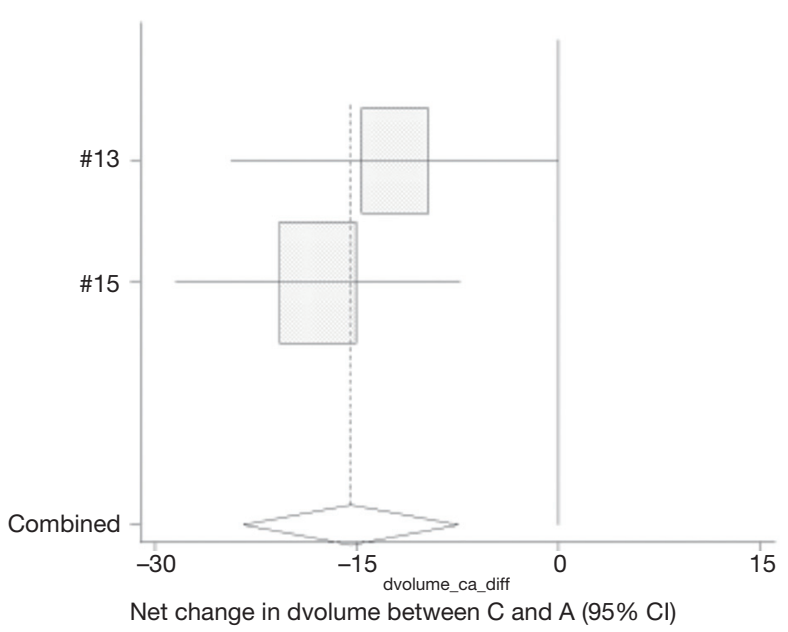

Figure 11 Effect on drain output volume with conventional vs. hemostatic gel.

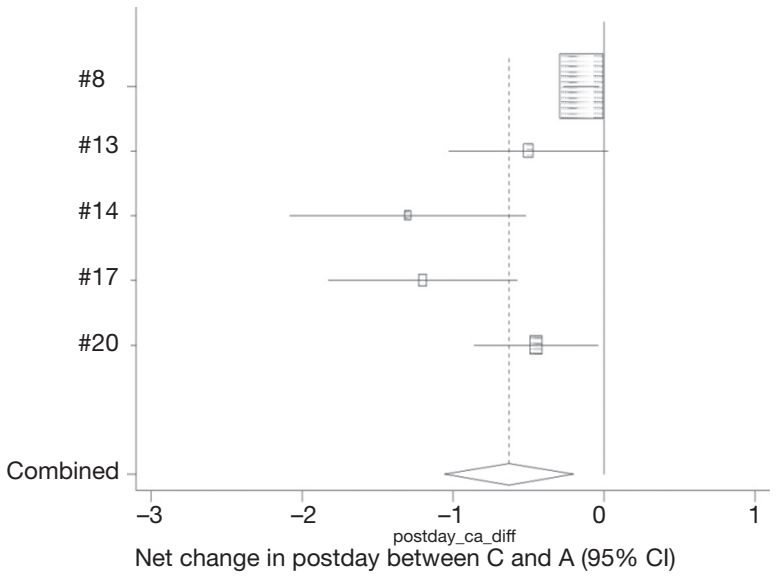

Figure 12 Effect on LOS with conventional vs. hemostatic agents.

hemostatic agents and conventional hemostasis.

\section{Hospital stay}

Five studies investigated the effect of hemostatic agents and conventional agents on length of hospital stay $(8,13,14,17,20)$. Patients who received hemostatic agents had shorter hospital stay when compared to the conventional group (95\% CI: $-1.057,-0.203$ ) (Figure 12). Three of the studies found that hemostatic agents had a significantly shorter hospital stay than conventional hemostasis $(14,17,20)$.

\section{Age}

Three studies evaluated the effect of age on the use of

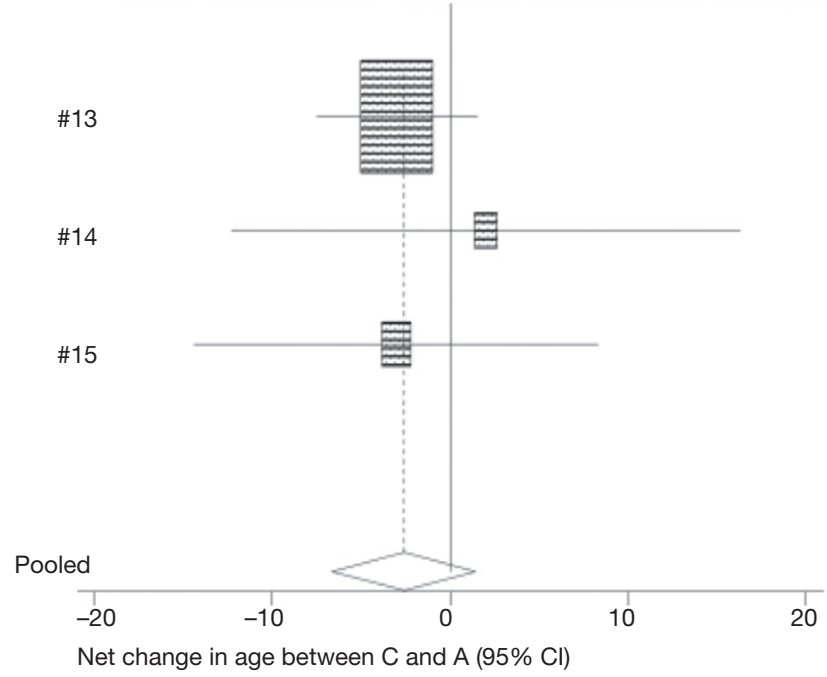

Figure 13 Effect of age on use of conventional vs. hemostatic agents.

hemostatic agents and conventional hemostasis (13-15). Although patients using hemostatic agents tended to be younger, there was no significant difference between the use of hemostatic agents and conventional hemostasis on age (95\% CI: -6.632, 1.412) (Figure 13). None of the studies found a significant difference with age.

\section{Discussion}

Due to the extensive vascular supply near the thyroid, intraoperative hemostasis is vital to avoid serious complications. Bleeding following thyroidectomies can result in several complications post-operatively leading to increased morbidity and mortality. Some of the complications that may result include hematomas, seromas, recurrent laryngeal nerve ischemia/dysfunction, infection, and hypoparathyroidism. These complications can result in increased recovery time and hospital stays. This metaanalysis of published articles intends to analyze the effects of hemostatic agents in thyroid surgery on preventing these complications as opposed to conventional hemostatic techniques. We found that while hemostatic agents did not significantly decrease the incidence of complications such as hematomas, seromas, infection, recurrent laryngeal nerve injury, or hypoparathyroidism, there was a significant decrease in operative time with the hemostatic patch and there was a significant decrease in drain output and hospital stay with the use of hemostatic agents. Future further studies are warranted to investigate the safety and 
effectiveness of hemostatic agents.

While hemostatic agents may have been found to decrease drain output, a few of the studies suggest that post-operative bleeding following thyroid surgery is more dependent on surgical skill than the use of intraoperative hemostatic agents $(8,9,13,19)$. However, they do suggest that hemostatic agents are a useful adjunct to good surgical technique. Additionally, a decrease in the duration a drain is in place may decrease the amount of post-operative discomfort that a patient may have (14). Some of the studies may not have taken into account the difficulty of certain operations or the amount of bleeding in each particular operation, which could obviously have an effect on complications resulting from the procedure. Uwiera et al. (20) found that there was a decrease in drainage with Tisseel whether a total thyroidectomy or a hemithyroidectomy was performed. Further control in surgical techniques in future studies compared to the use of hemostatic agents could aid in determining the efficacy of their use.

Several studies $(8,15)$ support the use of FloSeal as a first line hemostatic agent in thyroidectomy. Testini et al. (8) suggests that the increased cost in using FloSeal is offset by the resulting reduced time of operation and the decrease in hospital stay. Kim et al. (15) suggests their study may have been limited by the use of a single brand of FloSeal. They state that various brands and techniques in application of FloSeal may have an effect on the efficacy of FloSeal.

Moran (19) who studied the use of Surgicel patch relative to conventional hemostasis suggests that other hemostatic agents such as FloSeal and Tabotamp have yielded better results than Surgicel when observing the effects on operating time and duration of drain use. Testini (8) found that FloSeal was more effective than Tabotamp in reducing duration of drain use. The efficacy of the various hemostatic agents may have an effect on the results yielded and therefore further studies may be necessary to differentiate between the different types of hemostatic agents.

In conclusion, hemostatic agents appear to be a useful adjunct in achieving hemostasis in thyroidectomies. In this meta-analysis, hemostatic agents have been shown to decrease drain output and length of hospital stay following thyroidectomy. Further controlled studies are necessary to determine which hemostatic agents are more effective as well as whether hemostatic agents really have any significant advantage over conventional methods for hemostasis in preventing complications associated with thyroid surgery.

\section{Acknowledgements}

This research and work was fully supported by Tulane University Medical Center.

\section{Footnote}

Conflicts of Interest: The authors have no conflicts of interest to declare.

\section{References}

1. Gil Z, Patel SG. Surgery for thyroid cancer. Surg Oncol Clin N Am 2008;17:93-120, viii.

2. Harness JK, Fung L, Thompson NW, et al. Total thyroidectomy: complications and technique. World J Surg 1986;10:781-6.

3. Thompson NW, Olsen WR, Hoffman GL. The continuing development of the technique of thyroidectomy. Surgery 1973;73:913-27.

4. Zambudio AR, Rodríguez J, Riquelme J, et al. Prospective study of postoperative complications after total thyroidectomy for multinodular goiters by surgeons with experience in endocrine surgery. Ann Surg 2004;240:18-25.

5. Lacoste L, Gineste D, Karayan J, et al. Airway complications in thyroid surgery. Ann Otol Rhinol Laryngol 1993;102:441-6.

6. Harding J, Sebag F, Sierra M, et al. Thyroid surgery: postoperative hematoma--prevention and treatment. Langenbecks Arch Surg 2006;391:169-73.

7. Dionigi G, Boni L, Rovera F, et al. Dissection and hemostasis with hydroxilated polyvinyl acetal tampons in open thyroid surgery. Ann Surg Innov Res 2007;1:3.

8. Testini M, Marzaioli R, Lissidini G, et al. The effectiveness of FloSeal matrix hemostatic agent in thyroid surgery: a prospective, randomized, control study. Langenbecks Arch Surg 2009;394:837-42.

9. Lachachi F, Descottes B, Durand-Fontanier S, et al. The value of fibrin sealant in thyroid surgery without drainage. Int Surg 2000;85:344-6.

10. Spotnitz WD, Burks S. State-of-the-art review: Hemostats, sealants, and adhesives II: Update as well as how and when to use the components of the surgical toolbox. Clin Appl Thromb Hemost 2010;16:497-514.

11. Spotnitz WD, Burks S. Hemostats, sealants, and adhesives: components of the surgical toolbox. Transfusion 2008;48:1502-16. 
12. DerSimonian R, Laird N. Meta-analysis in clinical trials. Control Clin Trials 1986;7:177-88.

13. Guler M, Maralcan G, Kul S, et al. The efficacy of Ankaferd Blood Stopper for the management of bleeding following total thyroidectomy. J Invest Surg 2011;24:205-10.

14. Sözen S, Topuz O, Tükenmez M, et al. The use of fibrin sealant after total thyroidectomy for benign disease obviates the need for routine drainage. Results of a randomized controlled trial. Hippokratia 2011;15:247-51.

15. Kim TW, Choi SY, Jang MS, et al. Efficacy of fibrin sealant for drainage reduction in total thyroidectomy with bilateral central neck dissection. Otolaryngol Head Neck Surg 2012;147:654-60.

16. Ujam A, Awad Z, Wong G, et al. Safety trial of Floseal(®) haemostatic agent in head and neck surgery. Ann R Coll

Cite this article as: Khadra $\mathrm{H}$, Bakeer $\mathrm{M}$, Hauch A, Hu T, Kandil E. Hemostatic agent use in thyroid surgery: a metaanalysis. Gland Surg 2018;7(Suppl 1):S34-S41. doi: 10.21037/ gs.2018.03.02
Surg Engl 2012;94:336-9.

17. Matthews TW, Briant TD. The use of fibrin tissue glue in thyroid surgery: resource utilization implications. J Otolaryngol 1991;20:276-8.

18. Patel M, Garg R, Rice DH. Fibrin glue in thyroid and parathyroid surgery: is under-flap suction still necessary? Ear Nose Throat J 2006;85:530-2.

19. Amit M, Binenbaum Y, Cohen JT, et al. Effectiveness of an oxidized cellulose patch hemostatic agent in thyroid surgery: a prospective, randomized, controlled study. J Am Coll Surg 2013;217:221-5.

20. Uwiera TC, Uwiera RR, Seikaly H, et al. Tisseel and its effects on wound drainage post-thyroidectomy: prospective, randomized, blinded, controlled study. J Otolaryngol 2005;34:374-8. 